\title{
Growth Monitoring and Use of Growth Hormone in Children with Renal Failure
}

Hanna Lythgoe ${ }^{1}$, Emma O'Hagan ${ }^{1}$, Mohan Shenoy ${ }^{1}$

${ }^{1}$ Royal Manchester Children's Hospital

\section{Background}

- Chronic renal failure can cause significant growth impairment.

- Many factors contribute towards growth failure and it has a significant impact on morbidity, mortality and quality of life.

- Growth failure defined as $<2^{\text {nd }}$ centile for height

- $29 \%$ of children who undergo renal transplantation are $<2^{\text {nd }}$ centile for height

- $41 \%$ of children on dialysis are $<2^{\text {nd }}$ centile for height

- Patients who undergo renal transplantation experience some 'catch-up' growth but most patients do not reach their target height.

- It is important that growth is monitored regularly and growth failure addressed, including offering patients growth hormone $(\mathrm{GH})$ where appropriate.

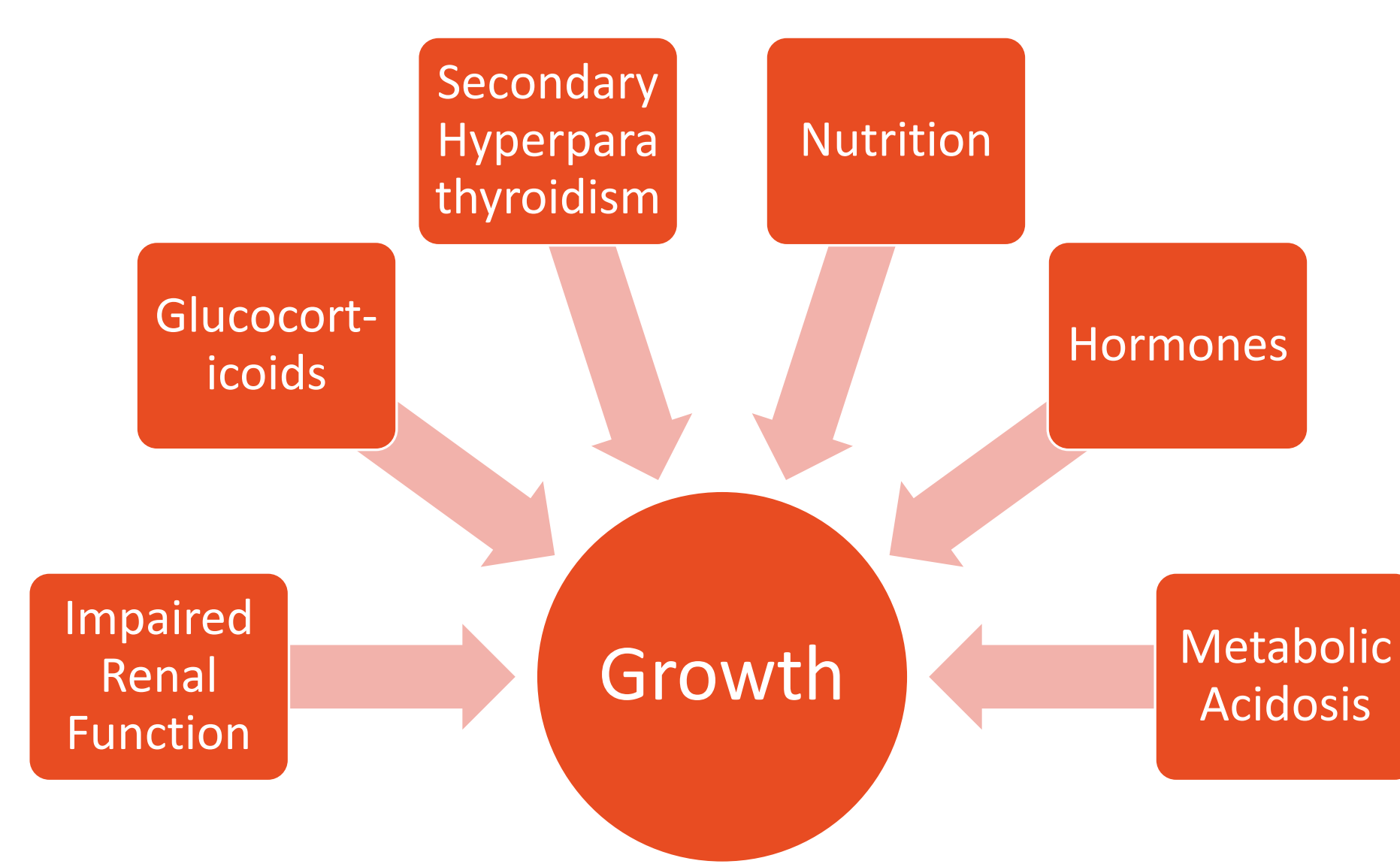

\section{Growth Hormone}

- Effects on short-term growth velocity (1 year) range from no improvement to approximately 1 standard deviation above the normal growth velocity

- Gains in final height range between $3-9 \mathrm{~cm}$

- Side effects include headache, paraesthesia, blurred vision, nausea and vomiting and injection site reactions

- Patients with chronic renal insufficiency or post transplantation are eligible if: height $<2^{\text {nd }}$ centile; no syndrome meaning GH deemed inappropriate; and parents and patient willing

\section{Aim}

We appraised our current practice to see how well we are monitoring growth in renal failure patients and if we are offering $\mathrm{GH}$ to eligible patients.

\section{Method}

We defined standards from NICE guidelines and Bristol guidelines endorsed by BSPED. We collected data from 76 patients on haemodialysis, peritoneal dialysis or post-renal transplant.

\section{Standards}

- Every child should have a growth chart in their current notes

- Every child should have growth measured and plotted at least twice a year

- All children with growth failure who are suitable should be offered growth hormone

- Growth hormone should be stopped if:

- growth velocity increases less than $50 \%$ from baseline in the first year of treatment

- final height is approached and growth velocity is less than $2 \mathrm{~cm}$ total growth in 1 year

- there are insurmountable problems with adherence

- final height is attained

\section{Results}

- $16 / 44$ (36\%) post-transplantation and $20 / 32(62 \%)$ dialysis patients had growth failure as defined by height $<2^{\text {nd }}$ centile, slightly higher than quoted in the literature $(29 \%$ and $41 \%$ respectively).

- 53/76 had growth charts in their notes, and 39/53 had their height and weight plotted regularly.

- 12 patients were potentially eligible for GH and not previously offered it.

- 11 patients were on or had previously received GH and were managed in line with NICE guidance.

\section{Conclusions}

- Growth monitoring needs to be improved in renal failure patients

- Growth failure should be highlighted in the problem list and addressed at clinic visits to improve their growth.

- 12 patients need discussing jointly by the nephrology and endocrinology teams and considering for $\mathrm{GH}$.

- Patients on or who have had GH are managed appropriately.

\section{Impact on Practice}

- 4 patients in the post-transplantation group (first group reviewed) have been offered GH and 2 families accepted it. 8 patients are currently being reviewed.

- Growth charts have been placed in all patient notes.

- Findings have been shared with the nephrology team in a local meeting to improve awareness. 